\title{
Altered mitochondrial oxidative phosphorylation capacity in horses suffering from polysaccharide storage myopathy
}

TOSI I. ${ }^{1}$, ART T. ${ }^{1}$, CASSART D. ${ }^{2}$, SERTEYN D. ${ }^{3}$ AND VOTION D. ${ }^{3}$

1 Center of Equine Sports Medicine, University of Liège, Liège, Belgium 2 Department of pathology and morphology, FVM, ULg, University of Liège, Liège, Belgium ${ }^{3}$ Equine clinic (FMV) \& Center of oxygen, research and development, ULg

Introduction: Polysaccharide storage myopathy (PSSM) is a widely described cause of exertional rhabdomyolysis that has been found in more than 35 equine breeds. Recent studies identified a dominantly genetic defect in the skeletal muscle glycogen synthase (GYS1) enzyme at the basis of the Type-1 PSSM phenotype (McCue et al., 2008). The condition is characterized by increased skeletal muscle glycogen concentration, and abnormal polysaccharide accumulation in myofibers. Gene expression studies (Barrey et al., 2009) indicated a down-regulation of some mitochondrial genes and we hypothesized that, in type-1 PSSM-affected horses, the energetic production through the oxidative phosphorylation (OXPHOS) in the mitochondria of myofibres might be impaired.

Our aim was to explore the muscle mitochondrial function of PSSM-affected horses using the High Resolution Respirometry, a sensitive diagnostic of the mitochondrial activity recently validated in the equine species (Votion et al., 2010).

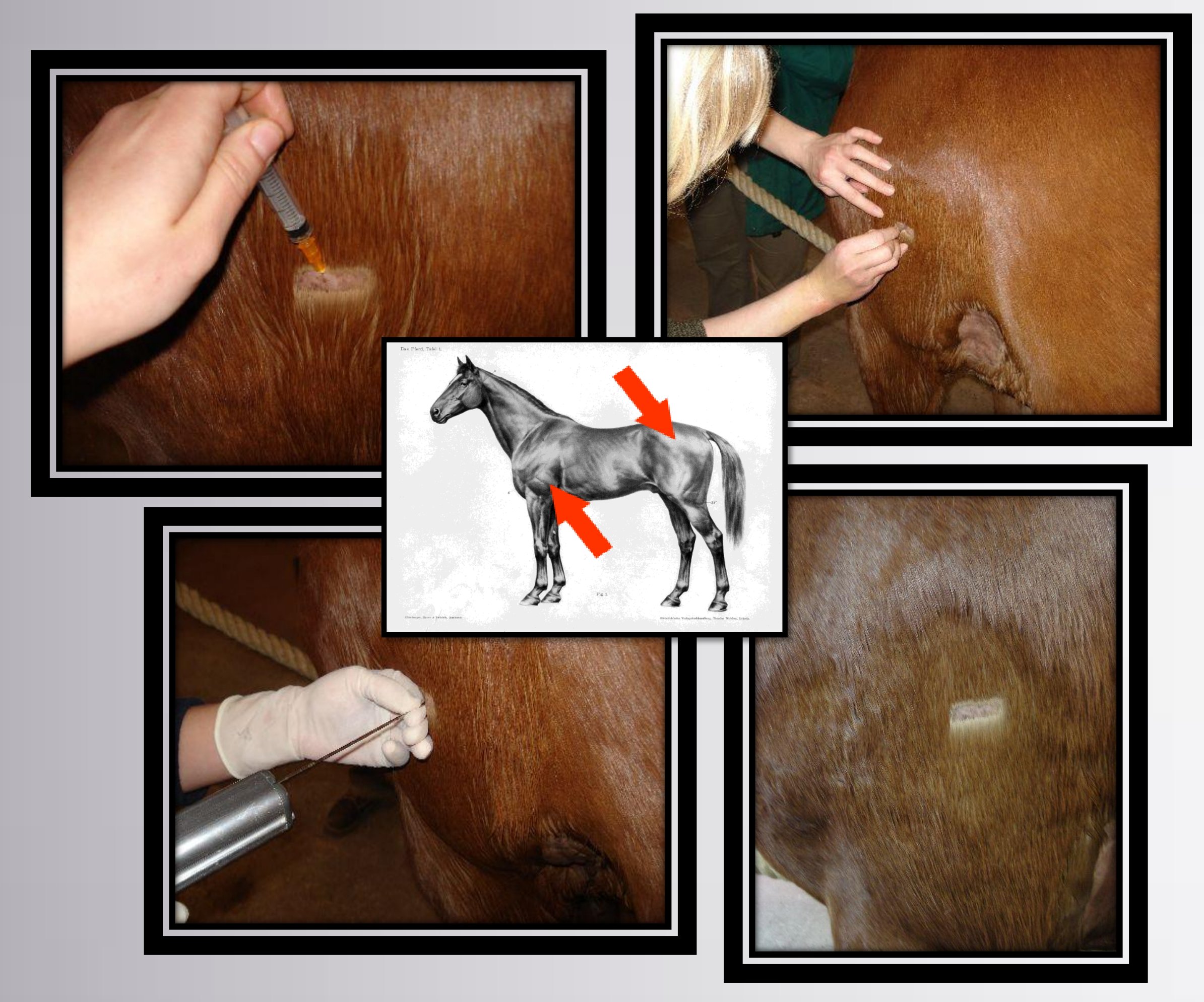

Fig.1. Muscle microbiopsy technique: An average $20 \mathrm{mg}$ of muscle tissue was obtained from both the $m$. triceps brachii and $m$. gluteus medius using a $14 \mathrm{G}$ biopsy needle mounted on an automatic instrument (Pro-MagTM, Angiotech, USA). Skin was shaved, desensitized and aseptically prepared. Muscle samples were immediately transferred to BIOPS, a preservation solution.

Results: Four horses were tested positive to type-1 PSSM (GYS1 mutation) at the genetic test and were included in the study. Histology revealed the presence of Periodic Acid Schiff (PAS)-positive accumulations of abnormal glycogen. A severe depression of the maximal oxidative phosphorylation capacity was observed (minus $38 \pm 14 \%$ ) by HRR, therefore confirming altered mitochondrial function in type-1 PSSM horses. Surprisingly, one PSSMpositive horse showed very high levels of mitochondrial respiration, even higher than control sport horses.

Fig.2. Gluteus medius muscle formalin fixed and paraffin embedded. Notice the presence of thick deposits of subsarcolemmal and intracytoplasmic glycogen (circles) with both the ematoxylin-eosin (A) and Periodic Acid Schiff staining (B)

\section{Materials and Methods:}

- Eight horses (mean age 8.1 - 4.4 y.o.), different breeds

- History of recurrent exertional rhabdomyolysis, exercise intolerance, increased serum CK/AST

- Specific complementary exams were performed to exclude other possible causes of exercise intolerance

- The presence of the GYS1 mutation was tested on each horse (EDTA whole blood sent to the Royal Veterinary College of London, England)

- Muscular biopsies were collected on the m. gluteus medius and m. triceps brachii muscles of each horse using the microbiopsy technique

- Histological analysis and high resolution respirometry (HRR) were realised on the muscle samples
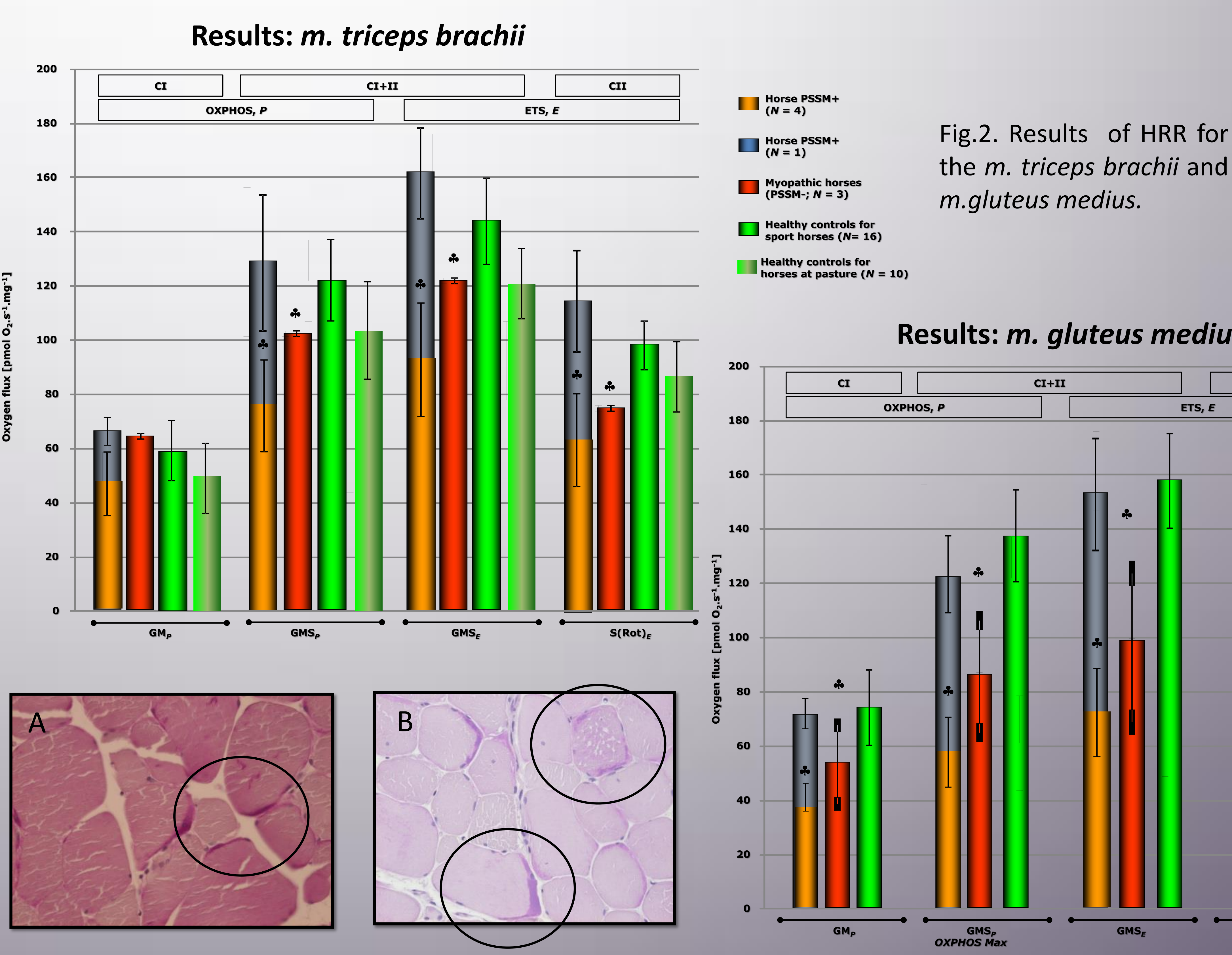

D $\begin{gathered}\text { Heathy controls for } \\ \text { sport horses }(N=16)\end{gathered}$

Healthy controls for
horses at pasture $(N=10)$

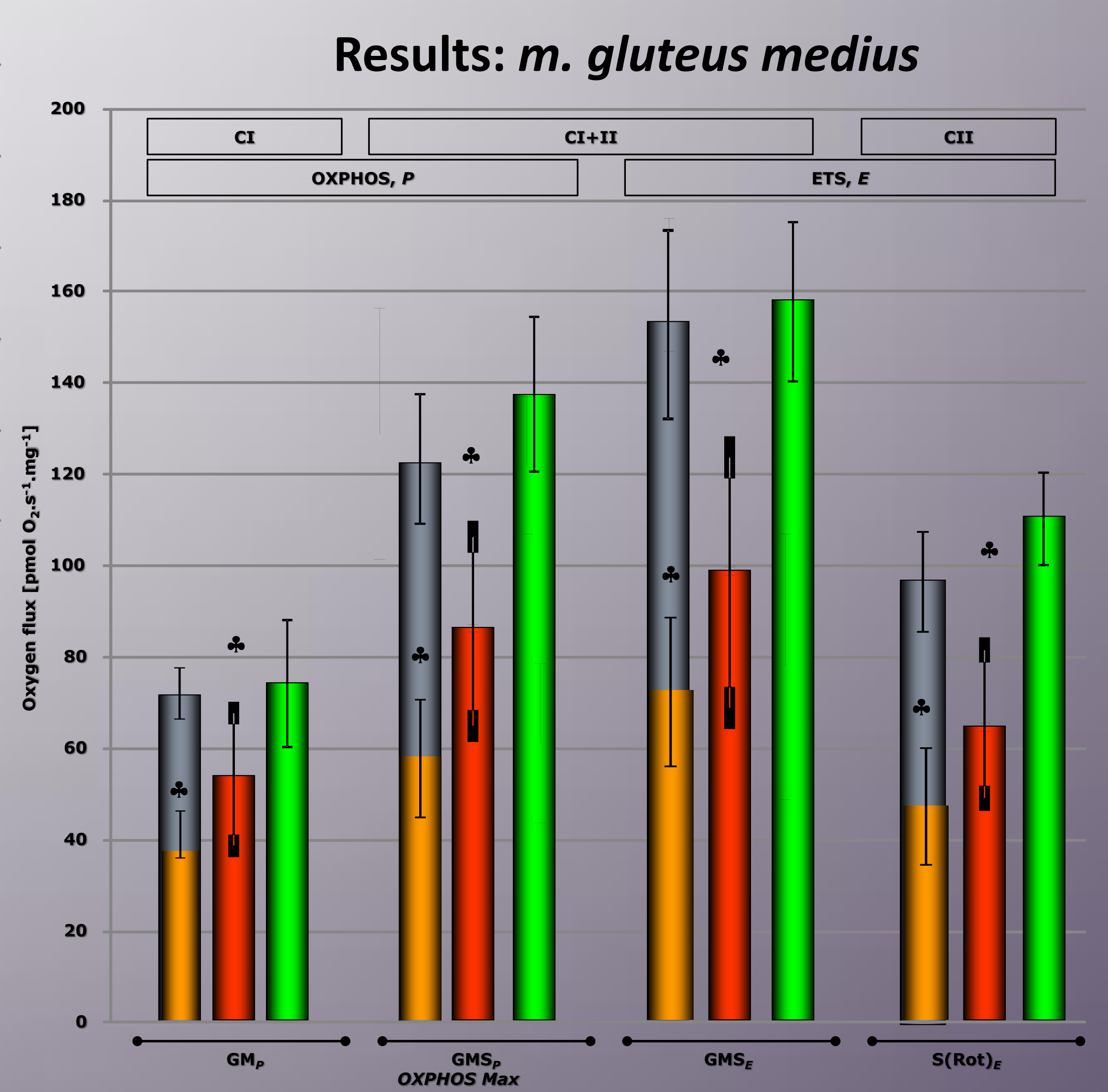

Conclusions

- All horses suffering from exertional rhabdomyolysis showed a severe decrease of mitochondrial respiration

- PSSM+ horses respiration was lower than PSSM- case

- In the $\boldsymbol{m}$. gluteus medius the decrease of mitochondrial respiration was more pronounced than in the $m$. triceps brachii

- Is there a possible mitochondrial recovery/compensatory response?

Equine PSSM is more than a glycogen storage disease? 